\title{
Endoscopic sinus surgery for foreign body extraction in an adult patient
}

Arianna Cardella ${ }^{1}$, Andrea Preti ${ }^{2}$, Roberto Gera ${ }^{3}$, Francesco Ottaviani ${ }^{4}$, and Francesco Mozzanica $^{4}$

${ }^{1}$ Università degli Studi di Milano

${ }^{2}$ University of Insubria Faculty of Medicine and Surgery

${ }^{3}$ IRCCS MultiMedica

${ }^{4}$ University of Milan Faculty of Medicine and Surgery

March 17, 2021

\begin{abstract}
The management of an unusual nasal foreign body is illustrated. A 34-year-old male presented to our outpatient clinic after inhalation of liquid cast during preparation of a plaster mask. The foreign body had solidified within the nasal cavities, causing obstruction and headache. Ambulatory removal was incomplete, therefore ESS was indicated.
\end{abstract}

\section{CLINICAL VIDEO}

TITLE: Endoscopic sinus surgery for foreign body extraction in an adult patient

Cardella $\mathrm{A}^{1,2}$, Preti $\mathrm{A}^{1,3}$, Gera $\mathrm{R}^{1}$, Ottaviani $\mathrm{F}^{1,2}$, Mozzanica $\mathrm{F}^{1,2}$

1 Department of Otorhinolaryngology, Ospedale San Giuseppe IRCCS Multimedica, Milan, Italy

2 Department of Clinical Sciences and Community Health, University of Milan, Milan, Italy

3 Department of Medicine and Surgery, University of Insubria, Varese, Italy

Corresponding author

Cardella Arianna, MD

Department of Clinical Sciences and Community Health, University of Milan, Milan, Italy

Via San Vittore 12, Milan, Italy

Email: arianna.cardella@unimi.it

Telephone: +390285994471

\section{ABSTRACT}

The management of an unusual nasal foreign body is illustrated. A 34-year-old male presented to our outpatient clinic after inhalation of liquid cast during preparation of a plaster mask. The foreign body had solidified within the nasal cavities, causing obstruction and headache. Ambulatory removal was incomplete, therefore ESS was indicated.

KEY CLINICAL MESSAGE 
Foreign bodies are an unusual indication for endoscopic sinus surgery. Whenever their extraction is not possible in the outpatient setting and acute sinusitis ensues, thorough exploration and extended dissection under general anesthesia should be taken into consideration in order to clear the nasal cavities.

\section{KEYWORDS}

Nasal foreign body - ESS - endoscopic sinus surgery

TEXT

Nasal foreign bodies are extremely common in the pediatric population while being an infrequent presentation for adults [1]. They are a predisposing factor for acute bacterial rhinosinusitis [2] and management of such infection cannot overlook the complete removal of the foreign body.

We illustrate the case of a 34-year-old male who developed nasal obstruction, headache and purulent rhinorrhea following the inhalation of liquid cast during preparation of a plaster mask. Complete ambulatory removal of the solidified cast was not possible, prompting a thorough dissection through endoscopic sinus surgery in order to clear all debris and explore the paranasal cavities.

\section{Conflict of interest}

Nothing to declare

\section{Funding}

Nothing to declare

\section{Autorship list}

- Arianna Cardella: substantial contribution to design of the study; drafting the manuscript; revising the manuscript for important intellectual content, final approval of the version to be published. Agreed to be accountable for all aspects of the work in ensuring that questions related to the accuracy or integrity of any part of the work are appropriately investigated and resolved.

- Andrea Preti: substantial contribution to design of the study; revising the manuscript for important intellectual content, final approval of the version to be published. Agreed to be accountable for all aspects of the work in ensuring that questions related to the accuracy or integrity of any part of the work are appropriately investigated and resolved

- Roberto Gera: substantial contribution to design of the study; revising the manuscript for important intellectual content, final approval of the version to be published. Agreed to be accountable for all aspects of the work in ensuring that questions related to the accuracy or integrity of any part of the work are appropriately investigated and resolved

- Francesco Ottaviani: substantial contribution to design of the study; revising the manuscript for important intellectual content, final approval of the version to be published. Agreed to be accountable for all aspects of the work in ensuring that questions related to the accuracy or integrity of any part of the work are appropriately investigated and resolved

- Francesco Mozzanica: substantial contribution to conception and design of the study; drafting the manuscript, final approval of the version to be published. Agreed to be accountable for all aspects of the work in ensuring that questions related to the accuracy or integrity of any part of the work are appropriately investigated and resolved.

References

M. Abou-Elfadl et al. European Annals of Otorhinolaryngology, Head and Neck diseases 132 (2015) 343-346

Fokkens WJ et al. European Position Paper on Rhinosinusitis and Nasal Polyps 2020 Rhinology. 2020 Suppl. 29: $1-464$.

Figure legend 
Figure 1: Intraoperative view of the foreign body

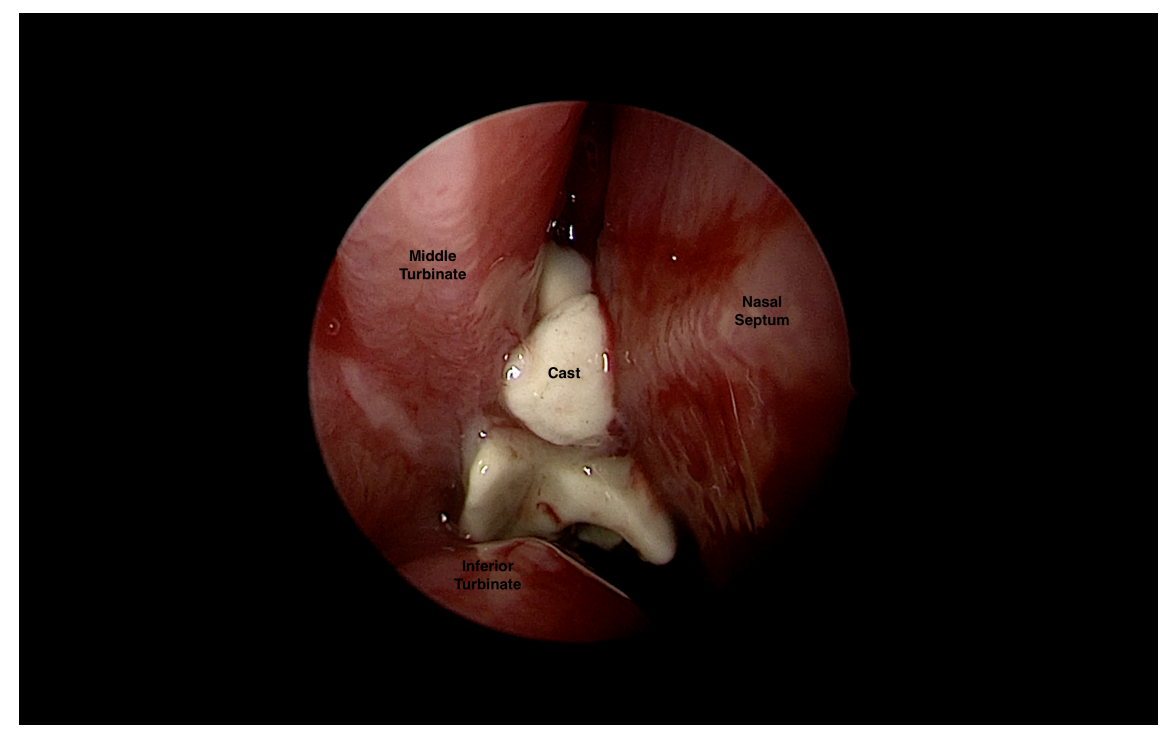

